\title{
Recent Goldstone radar observations of selected near-Earth asteroids less than $140 \mathrm{~m}$ in diameter
}

\author{
Marina Brozović ${ }^{1}$, Lance A. M. Benner ${ }^{1}$, Michael W. Busch ${ }^{2}$, \\ Jon D. Giorgini ${ }^{1}$, Martin A. Slade ${ }^{1}$ and Kenneth J. Lawrence ${ }^{1}$ \\ ${ }^{1}$ Jet Propulsion Laboratory, California Institute of Technology \\ Pasadena, CA 91109, USA \\ email: Marina.Brozovic@jpl.nasa.gov \\ ${ }^{2}$ SETI Institute \\ Mountain View, CA 94043, USA
}

\begin{abstract}
Radar observations are a powerful technique to study near-Earth asteroids (NEAs). Goldstone's $3.75 \mathrm{~m}$ resolution capability is invaluable when attempting to image NEAs with diameters smaller than $140 \mathrm{~m}$. The small NEAs are a very diverse population in which we continue to discover unusual objects.
\end{abstract}

Keywords. techniques: radar astronomy; minor planets, asteroids

\section{Introduction}

The number of near-Earth asteroids ranging between few meters to 140 meters in diameter is estimated to be in the millions, but less than one percent has been discovered (Mainzer et al. 2015). How are the characteristics of these small objects different from the properties of asteroids that have diameters in hundreds of meters or several kilometers? What are their shapes, spins, compositions, interiors, and origins? This paper gives a brief overview of recent findings with the Goldstone Solar System Radar (GSSR). All the statistics and other numbers quoted here are based on Goldstone observations only.

1998 KY26 (Ostro et al. 1999) was the first object in this category observed with radar at Goldstone, and to date almost 50 have been detected. Since 2012, small NEAs account for $1 / 3$ of the $\sim 30$ NEAs observed annually at Goldstone. $90 \%$ of NEA radar targets with $H>22$ are targets-of-opportunity observed within days or weeks of discovery. Objects this small are detectable by radar only within about ten lunar distances or less. Radar astrometry is particularly important for these asteroids because they are too faint to be followed for long with optical telescopes. A radar range measurement often secures their orbit for decades or centuries, where otherwise the object would be lost and require rediscovery. For single apparition cases, radar extends the time-span of Earth encounter prediction by factor of five on average (Ostro \& Giorgini 2004).

\section{Radar Observations}

We use three setups in radar experiments at Goldstone: continuous wave $(\mathrm{CW})$, binary phase coded (BPC) waveforms, and linear frequency modulation (LFM) or chirp waveforms. During CW observations, we transmit a circularly polarized electromagnetic wave of constant amplitude and frequency. The wave reflects off the surface and the targets rotation spreads the signal in Doppler frequency. The Doppler broadening of the echo is a 
function of the rotation period, the diameter of the asteroid, and the spin axis alignment:

$$
B=\frac{4 \pi D}{\lambda P \cos \delta}
$$

where $\mathrm{B}$ is the bandwidth, $\mathrm{P}$ is the rotation period, $\mathrm{D}$ is the objects diameter, $\lambda$ is the radar wavelength $(3.5 \mathrm{~cm}$ at Goldstone), and $\delta$ is the subradar latitude.

The reflected wave can return in the same sense of circular polarization (SC) as the outgoing beam, or in the opposite sense (OC). Echoes from a smooth surface at decimeter scales will return almost entirely in the OC channel. SC echoes can result from multiple scattering from rough surfaces, single scattering from surfaces with radii of curvature comparable to the radar wavelength, and from coherent backscattering. The ratio of the two echoes, SC/OC, represents a gauge of the targets near-surface roughness. CW observations are often the only mode of detection of NEAs with $H>22$ because many have low SNRs at Goldstone. CW data provide insight into a rotation period, elongation, convex hull shape, pole direction, cross section, radar albedo, and a circular polarization ratio.

Binary phase coded and chirp waveforms are used for ranging and delay-Doppler imaging. In binary phase coded observations the transmitted signal is phase-modulated via a repeating pseudo-random binary code. We use this mode of operation in order to achieve resolutions up to $15 \mathrm{~m}$ at Goldstone. The chirping setup uses linear frequency modulation (Slade et al. 2011; Quirk \& Srinivasan 2013) as an alternative way to modulate the carrier wave. This method allows us to utilize the full bandwidth of Goldstones klystron amplifiers $(40 \mathrm{MHz})$ without violating our frequency license. Chirping at $40 \mathrm{MHz}$ chirp gives a range resolution of 3.75 meters. This high-resolution capability is relatively new and it has allowed radar imaging of about a dozen objects with diameters between 30-140 $\mathrm{m}$ since its inception in 2010. 2010 AL30 was the first NEA to be imaged with $3.75 \mathrm{~m}$ resolution in January of 2010 (Slade et al. 2010).

Radar observations of objects that are closer than $\sim 4$ lunar distances (corresponding to a distance of 0.01 au and a $\sim 10.3$ second radar signal round-trip-time, RTT) previously required coordination between two telescopes, one to transmit and one to receive, due to the short RTT and need to physically switch between transmit and receive configurations. However, the switching process has been accelerated and observations since December 2013 have shown that Goldstone can now conduct monostatic observations with RTTs of $\sim 5$ seconds which has simplified logistics with numerous targets that are between 0.005-0.01 au from Earth. We schedule bistatic reception at Green Bank or Arecibo because these configurations increase the SNRs by factors of two and five due to the larger receiving antennas and yield delay-Doppler images with higher resolutions than are possible at Goldstone. Bistatic observations also allow for better Doppler frequency resolution. In monostatic observations, we can only resolve the object in Doppler frequency up to $1 / \mathrm{RTT} \mathrm{Hz}$. In bistatic observations, the Doppler frequency resolution is an inverse of the time of integration, so the longer we integrate, the finer the frequency resolution. For short RTTs, we also often receive DSS-14 transmissions at DSS-13, a $34 \mathrm{~m}$ antenna at the Goldstone complex. DSS-13 is easier to schedule on a short notice than Arecibo or GBT, but bistatic SNRs at DSS-13 are only 1/6 of the signal strength at DSS-14 alone.

As of late 2014, DSS-13 has its own radar capability in a form of $80 \mathrm{~kW}, 80 \mathrm{MHz}$ bandwidth klystron. DSS-13 is more than an order of magnitude less sensitive than the $70 \mathrm{~m}$ DSS-14, but for targets with strong SNRs, DSS-13 can obtain images with a resolution of $1.875 \mathrm{~m}$ that is twice as fine as the highest resolution at DSS-14 and four times as fine as the highest resolution at Arecibo. The radar at DSS-13 has been 
successfully tested at 3.75 m resolution with (357439) 2004 BL86 (Benner et al. 2015) and 2015 HM10 (Busch et al. 2015). With both asteroids, the echoes were received at the Green Bank Telescope. This new radar capability, as well as the recent increase in observing time at Goldstone will yield more high-resolution imaging opportunities of small NEAs.

\section{Shapes of NEAs with $H>22$}

Resolving the shapes of small asteroids is challenging even with the $3.75 \mathrm{~m}$ resolution that is now available at Goldstone. About a dozen small NEAs have been imaged and resolved at that resolution since 2010, and it appears that at least $70 \%$ are irregular in shape with significant elongation. There are also a few objects such as 2012 BX34, 2013 FB8, and 2014 BX34 that appear spheroidal despite their small sizes. In contrast with larger NEAs, we have not detected any binary or contact binary systems among NEAs with $H>22$.

\subsection{BV15}

2013 BV15 approached within 0.0095 au (3.7 lunar distances) on February 13, 2013, less than one month after it was discovered by Pan-STARRS 1 (M.P.E.C. 2013-B28). The asteroid was too far south for Goldstone to observe during the closest approach, but we scheduled two radar tracks on February 11 and 12, while the asteroid was still inbound. Nothing was known about this object prior to the radar observations except its absolute magnitude, $\mathrm{H}=24.3$ suggested a diameter within a factor of two of 45 meters. Lightcurves obtained by Carl Hergenrother (pers. comm.) indicated a rotation period of 0.48 hours and an amplitude of $0.7 \mathrm{mag}$. Figure 1 shows a collage of delay-Doppler images of 2013 BV15. The images demonstrate that 2013 BV15 is an irregular, elongated object with at least one distinct concavity. A preliminary estimate of the pole-on dimensions is $\sim 60 \times$ $30 \mathrm{~m}$. The rotation of the object in the images is consistent with the period estimated from the lightcurves. The radar astrometry prevented 2013 BV15 from being lost and the Earth encounter predictability window was extended from 2013 apparition only to 154 years $(1912-2066)$.

\subsection{ET}

We observed 2013 ET on March 10, 2013, less than one week after its discovery by the Catalina Sky Survey (M.P.E.C. 2013-E14). Its absolute magnitude of 23.1 suggested a diameter within a factor of two of $\sim 75 \mathrm{~m}$, and Joe Pollock (pers. comm.) reported a $\sim 2 \mathrm{~h}$ rotation period and evidence for non-principal axis rotation based on lightcurves obtained at Panchromatic Robotic Optical Monitoring and Polarimetry Telescopes (PROMPT). The lightcurves also showed a considerable magnitude variation of $\sim 1$ mag that suggested an elongated shape. Figure 2 shows delay-Doppler images that reveal an irregularly shaped object with complex surface topography. In certain orientations, we see radar-bright lines of pixels parallel to the leading edge. These give the impression of grooves, ridges, or layering on the asteroid. This is just a visual description as opposed to a statement about a possible physical mechanism that shaped the asteroid. Similar features were observed in recent radar imaging of 2015 HM10 (Busch et al. 2015), which is an irregular object with pole-on dimensions of $\sim 80 \times 40 \mathrm{~m}$. Our estimate for the pole-on dimensions of $2013 \mathrm{ET}$ is $70 \times 40 \mathrm{~m}$, but the data coverage is not sufficient for shape modeling and spin state estimation. 


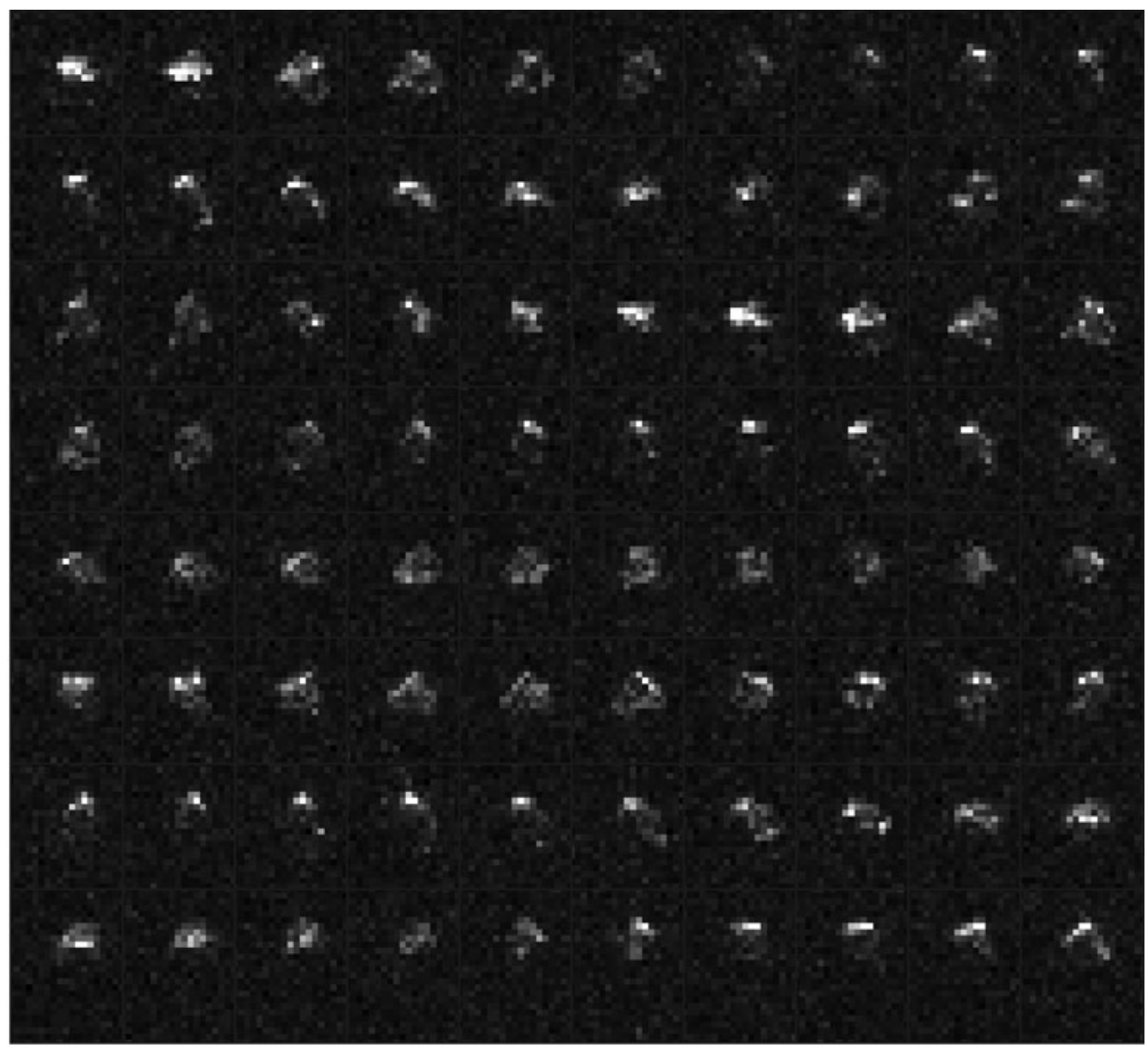

Figure 1. Sequence of 2013 BV15 radar images obtained on February 12, 2013 between 08:29-09:59 UTC. Delay-Doppler resolution is $3.75 \mathrm{~m} \times 1.0 \mathrm{~Hz}$. Each frame is a weighted sum of 3 receiving cycles that span $\sim 53 \mathrm{sec}$. Time starts at the upper left corner, increases to the right, and ends at the bottom right. Range increases downward in each frame and Doppler frequency increases to the right, so rotation is counterclockwise. The entire sequence is close to 90 minutes long and it covers about three rotations by the asteroid.

\section{Monoliths vs. rubble piles in $H>22$ NEA population}

Pravec \& Harris (2000) were the first to analyze the distribution of (mostly nearEarth) asteroid spin rates and their sizes, and they have concluded that the lack of objects rotating faster than the $2.2 \mathrm{~h}$ period among asteroids larger than a few hundred meters, as well as the tendency to spheroidal shapes of fast rotators is evidence that asteroids larger than a few hundred meters are mostly loosely bound, gravity-dominated aggregates with negligible tensile strength (rubble piles), while monoliths may be abundant among smaller objects. This statement assumes that rubble piles have no cohesive strength between their components, and that only monolithic objects can sustain rapid rotation rates. Brown, Pack, Edwards, et al. (2004); Scheeres et al. (2010); Sánchez \& Scheeres (2014) have discussed how even small asteroids that rotate with periods of less than an hour could be bound aggregates (or quasi-rubble piles) held together by cohesive forces. Brown, Pack, Edwards, et al. (2004) noted that centimeter-sized grains in proximity to each other can provide sufficient cohesive force to withstand a few-minute period 


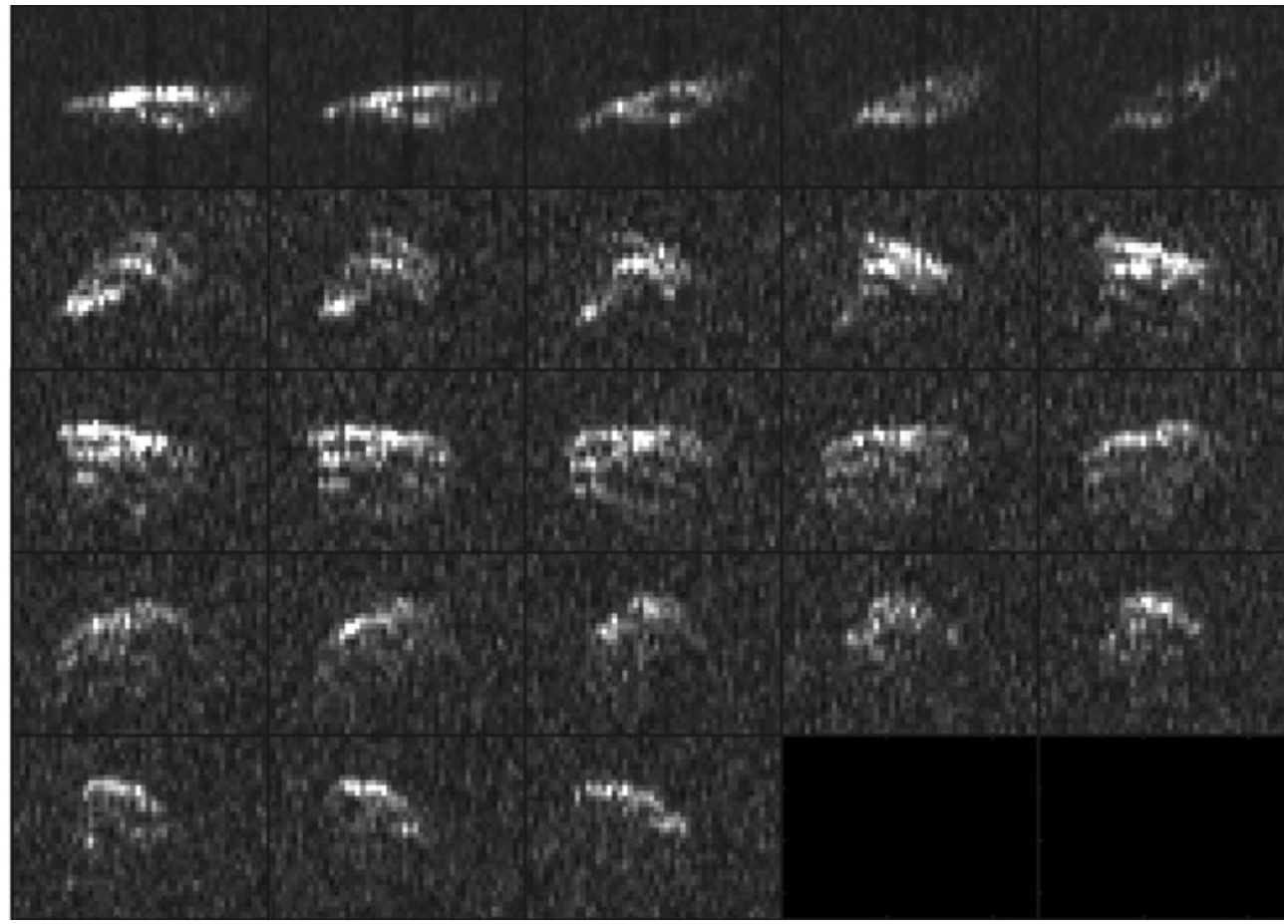

Figure 2. A sequence of 2013 ET radar images obtained on March 10, 2013 between 01:13-02:50 UTC. Each frame has a resolution of $1.875 \mathrm{~m} \times 0.015 \mathrm{~Hz}$, and is an integration of $240 \mathrm{~s}$. The data were obtained using $40 \mathrm{MHz}$ chirps (3.75 m range resolution) that were double sampled to give $1.875 \mathrm{~m} / \mathrm{row}$. The vertical lines in the first five frames are due to echo overlapping the DC line at $0 \mathrm{~Hz}$ Doppler. We have later put an offset of $+10 \mathrm{~Hz}$ to move the echo away from the carrier frequency.

rotation rate of a $100 \mathrm{~m}$ asteroid. For a $10 \mathrm{~m}$ body, similar grain sizes could withstand a rotation period of less than one minute. Brown, Pack, Edwards, et al. (2004) suggested the ultimate strength of a small aggregate is determined perhaps by shock fractures or other imperfections. Sánchez \& Scheeres (2014) noted that the population of rapidly rotating asteroids could consist of both distributions of smaller grains (i.e., rubble piles) and of monolithic boulders.

\subsection{BR57}

2014 BR57 was a target-of-opportunity in February of 2014, and we observed it 3 weeks after its discovery by Mt. Lemon Survey (M.P.E.C. 2014-B80). Delay-Doppler images (Figure 3) show a rounded object that has a visible extent, an approximation of its radius, of $\sim 40 \mathrm{~m}$. The radar data suggest that the rotation period is $\sim 4-5$ hours; so, this asteroid is not a rapid rotator. Images obtained at a range resolution of $3.75 \mathrm{~m}$ reveal radar-bright pixels that remain visible from frame to frame and have a trajectory consistent with the delay-Doppler projection of a surface feature rotating with the asteroid (i.e. similar to Figure 1-13 in Evans \& Hagfors (1968)). The spots appear in frames 5, 6, and 7 at the trailing edge of the echo and in frames 11, 12, and 13 near the two o'clock position. We interpret these features as candidates for surface boulders. Busch et al. (2015) also report on a possible boulder candidate on 2015 HM10. 2014 BR57 and 2015 HM10 are the smallest known NEA with radar evidence for boulders, and 2014 BR57s rounded appearance resembles those of larger objects thought to be rubble piles. 


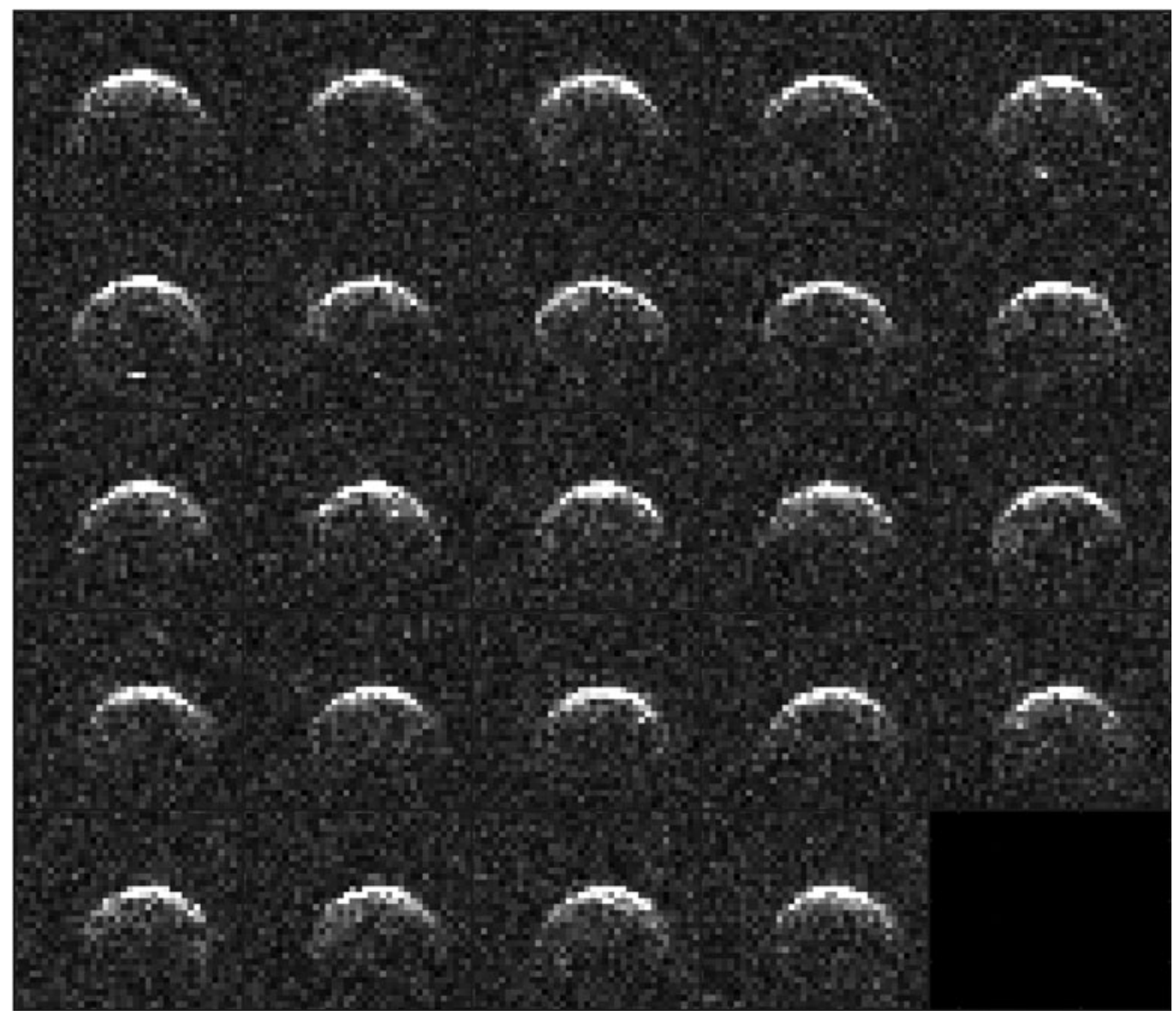

Figure 3. A sequence of 2014 BR57 radar images obtained on February 182014 between 03:01-07:14 UTC. Each image is a weighted sum of 15 receiving cycles that span 10 minutes. The resolution is $3.75 \mathrm{~m} \times 0.06 \mathrm{~Hz}$.

Radar astrometry obtained on February 18 permits reliable orbit estimation for 261 years (1946-2207), and has prevented 2014 BR57 from being lost. The encounter in 2014 was the closest for the foreseeable future.

\section{Rapid rotation of small NEAs}

Rotation periods for small NEAs range from only 16 s for 2014 RC (Thirouin, Skiff, and Moskovitz, pers. comm.) to weeks for 2001 EC16 (Alan W. Harris, pers. comm., Jean-Luc Margot, pers. comm.). Goldstone has observed close to 50 NEAs with $H>22$ to date, and the data are sufficient for first-order determination of their periods. The periods were estimated either from Eq. 2.1 or from progression of surface features in high-resolution imaging. We also take into account the uncertainties in size and sub-radar latitude. The radar-derived periods are cross-checked with periods obtained from photometry if they are available. Our preliminary conclusion is that more than $75 \%$ of NEAs with $H>22$ have rotation periods of less than one hour.

\section{1. $2014 R C$}

The ultra-rapid rotator $2014 \mathrm{RC}$ was observed by Goldstone on September 7, 2014. At the time of observations, the asteroid was just over one lunar distance from Earth. The 


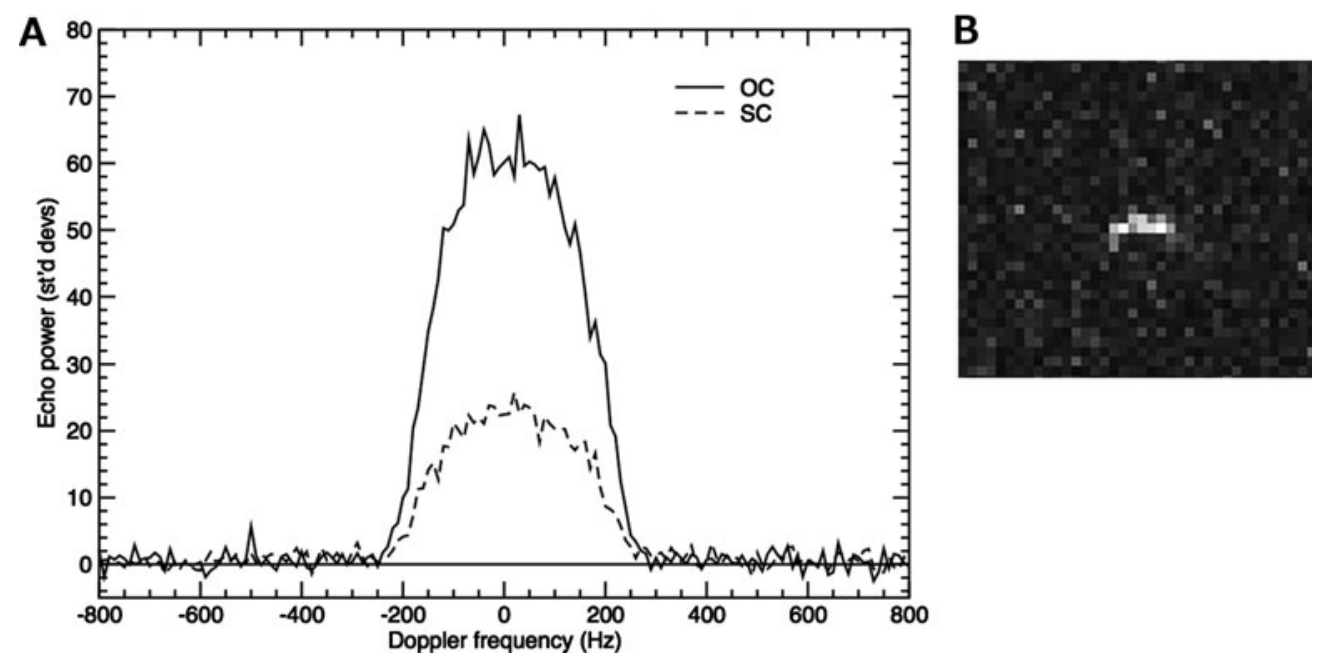

Figure 4. A. Echo power spectrum of 2014 RC obtained on September 7, 2014 between 07:05-07:42 UTC. DSS-14 was transmitting and DSS-13 was receiving. The Doppler resolution is $10 \mathrm{~Hz}$. The solid and dashed lines show the detections in the OC and SC channels of circular polarization. B. Bistatic delay-Doppler image of 2014 RC from September 7, 2014. Resolution is $3.75 \mathrm{~m} \times 50 \mathrm{~Hz}$. This radar image was produced from a 16-second integration that spans one full rotation by the asteroid. The asteroid rotates so rapidly and it is so small that the radar images do not show any details.

Doppler bandwidth (Figure 4 ) for this object was $\sim 500 \mathrm{~Hz}$. If we combine the measured $500 \mathrm{~Hz}$ Doppler bandwidth with a 16 sec rotation period from the lightcurves (Thirouin, Skiff, and Moskovitz, pers. comm.), and assume radar view perpendicular to the spin axis, then we obtain a diameter estimate of $\sim 20 \mathrm{~m}$. This was confirmed in $3.75 \mathrm{~m}$ resolution delay-Doppler image (Figure 4) where we integrated the signal over one full rotation, and obtained a visible extent of $\sim 7.5-11 \mathrm{~m}$. Assuming a symmetric object, the diameter should be double the visible extent.

\section{NHATs targets}

Some of the objects with $H>22$ could be destinations for future manned and/or robotic missions. Goldstone has observed more than 20 small asteroids on the NearEarth object Human spaceflight Accessible Targets Study (NHATS†) list to date and below we briefly discuss radar observations of two of them.

\subsection{RH120}

2006 RH120 was discovered by the Catalina Sky Survey in September of 2006, but it only got its minor planet designation in 2008. Up to that time, it carried Catalina Sky Survey catalog designation: 6R10DB9. This object has an absolute magnitude H of 29.5 suggesting a diameter of a few meters. The orbit of 2006 RH120 is an extreme case of an Earth-like orbit because the asteroid gets temporarily captured in the Earth-Moon system every $\sim 20$ years. 2006 RH120 is the only known member of the population of "mini-moons" (Bolin et al., 2014).

Kwiatkowski et al. (2009) observed 2006 RH120 from the 10-m South African Large Telescope on several nights in March of 2007. The lightcurves revealed a rapid rotation

$$
\dagger \text { http://neo.jpl.nasa.gov/nhats/ }
$$


period of 2.75 minutes and large amplitude of $1.2 \mathrm{mag}$. These results indicated that the asteroid could be a monolith and that it is likely quite elongated.

We observed 2006 RH120 at Goldstone on June 12, 14, and 17, 2007 while the asteroid was making its fourth and final revolution about Earth. The asteroid approached within one lunar distance at this time; so, we scheduled a bistatic experiments with DSS-14 transmitting and DSS-13 receiving on June 12 and 14, and, due to a scheduling conflict, DSS-25 receiving on June 17 . Goldstone CW observations spanned several hours on each day and covered well over 100 rotations by 2006 RH120. A CW echo power spectrum is shown in Figure 5. Benner et al. (2008) reported a circular polarization ratio, $S C / O C=$ $0.58 \pm 0.03$. The maximum observed bandwidth was $\sim 8 \mathrm{~Hz}$. The combination of maximum bandwidth and the rotation period gives a constraint for the long axis size: $D_{L}=$ $3.7 \mathrm{~m} / \cos \delta$.

Prior to the radar observations, the question was raised if this small asteroid could be old flight hardware or possibly material ejected off the Moon by an impact. A preliminary analysis done by Paul W. Chodas at the Jet Propulsion Laboratory (JPL) showed that the magnitude of the detected solar radiation pressure perturbations was consistent with expectations for a rocky, natural object, but not with flight hardware (P.W. Chodas, pers. comm.). The high value of SC/OC from the radar observations suggests significant near-surface roughness at centimeter scales and it rules out flight hardware such as an old rocket upper stage, which would not be rough. Radar constraint on the size of 2006 RH120 also suggests that the object cannot be an old rocket upper stage because it's too small. Goldstone radar data will yield an estimation of the convex hull shape in the future and shed more light on (natural) origin of this object.

\subsection{BS45}

2013 BS45 was discovered by Spacewatch on January 20, 2013 (M.P.E.C. 2013-B72), and we observed it from Goldstone on February 10, 12, and 13 when it was 0.013 au $(\sim 4.9$ lunar distances) away. This asteroid is in a very Earth-like orbit that makes it an excellent NHATs target. When viewed in an Earth-rotating frame, the asteroid's orbit appears to be tracing a horseshoe shape with the Earth between the "horns" (P.W. Chodas, pers. comm.). Several such objects have already been discovered in the near-Earth population: 54509 YORP (2000 PH5), 2002 AA29, and 2010 SO16 (Connors et al. 2002; Wiegert et al. 2002; Margot \& Nicholson 2003; Brasser et al. 2004; Christou \& Asher 2011).

2013 BS45 has an absolute magnitude of 25.7, which places an upper bound (assuming an optical albedo of $3 \%$ ) on its diameter of $\sim 50 \mathrm{~m}$. 2013 BS45 was not a very strong target at Goldstone, but we obtained some $\mathrm{CW}$ and coarse-imaging data. The $\mathrm{CW}$ echo power spectrum in Figure 5 revealed a wide Doppler bandwidth of $\sim 180 \mathrm{~Hz}$, which is an indication of the objects rapid rotation. The $\sim 50 \mathrm{~m}$ size constraint was tested with 30 minutes of 0.125 us (19 m) delay-Doppler imaging. The echo of 2013 BS45 was 2 range pixels deep, corresponding to $38 \mathrm{~m}$, constraining the diameter to at most $76 \mathrm{~m}$. Equation 2.1 and estimates of 2014 BS45's size and bandwidth suggest a rotation period of a few minutes that could mean that this object is a monolith. Radar astrometry has extended the interval of reliable orbit predictability by $\sim 155$ years.

\section{Conclusions}

Goldstone's $3.75 \mathrm{~m}$ imaging capability at DSS-14 has proven invaluable in characterizing NEA with $H>22$. The recent increase in observing time at Goldstone and the new radar capability at DSS-13 at the Goldstone complex will yield images of more objects in this size class. Characterizing this population is important for scientific assessment 

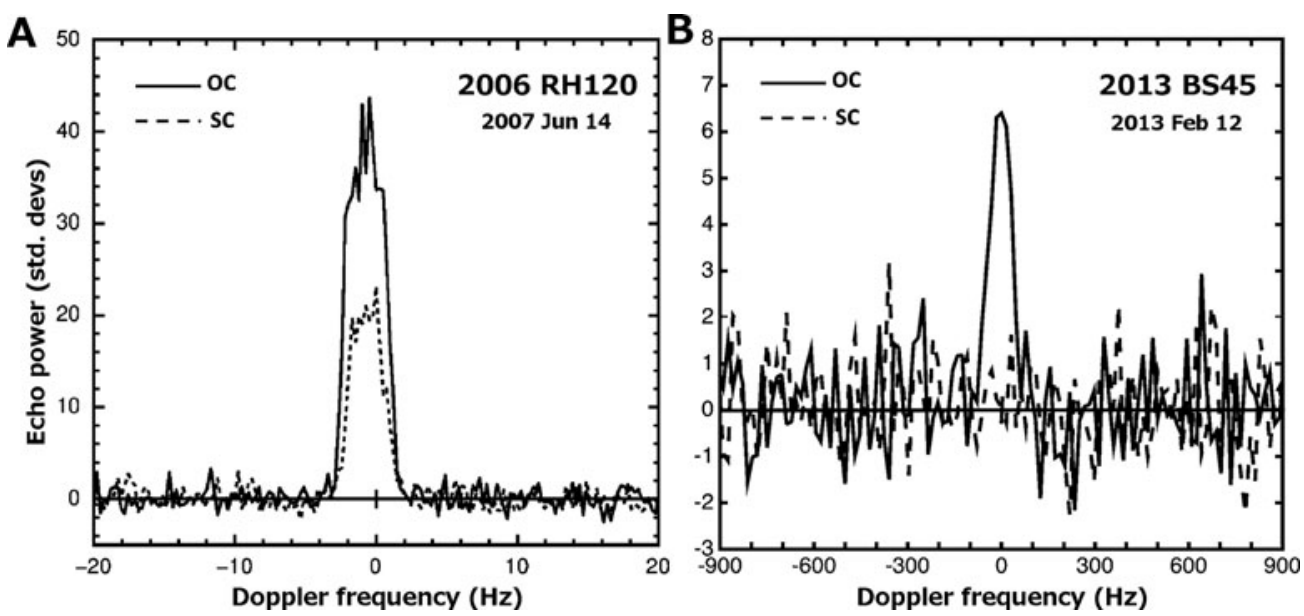

Figure 5. A. Echo power spectrum of 2006 RH120. 2006 RH120 was observed on June 14, 2007 from 04:30-05:00 UTC in a bistatic mode where DSS-14 was transmitting and DSS-13 was receiving. Doppler resolution is $0.24 \mathrm{~Hz}$. B. Echo power spectrum of 2013 BS45. The asteroid was observed on February 12, 2013 from 10:18-10:31 UTC. Doppler resolution is $15.6 \mathrm{~Hz}$.

and because some of the objects could be destinations for future manned and/or robotic missions.

\section{Acknowledgements}

We would like to thank Petr Pravec for reviewing this manuscript. We also thank the Goldstone technical and support staffs for help with the radar observations. This research was conducted at the Jet Propulsion Laboratory, California Institute of Technology under a contract with NASA.

\section{References}

Benner, L. A. M., Ostro, S. J., Magri, C., Nolan, M. C., Howell, E. S., Giorgini, J. D., Jurgens, R. F., Margot, J.-L.,Taylor, P. A., Busch, M. W., \& Shepard, M. K. 2008, Icarus, 198, 294 Benner, L. A. M., Brozovic, M., Giorgini, J. D., Taylor, P. A., Howell, E. S., Busch, M. W., Nolan, M. C., Jao, J. S., Lee, C. G., Slade, M. A., Ford, H. A., \& Ghigo, F. D. 2015, IAU General Assembly, 22, 57549

Bolin, B., Jedicke, R., Granvik, M., Brown, P., Howell, E., Nolan, M. C., Jenniskens, P., Chyba, M., Patterson, G., \& Wainscoat, R., 2014, Icarus, 241, 280

Brasser, R., Innanen, K. A., Connors, M., Veillet, C., Wiegert, P., \& Mikkola, Seppo, Chodas, P. W. 2004, Icarus, 171, 102

Brown, P., Pack, D., Edwards, W. N., Revelle, D. O., Yoo, B. B., Spalding, R. E., \& Tagliaferri, E., 2004, Meteoritics \& Planetary Science, 39, 1781

Busch, M. W., Benner, L. A. M., Naidu, S. P., Brozovic, M., Richardson, J. E., Rivera-Valentin, E. G., Taylor, P. A., Ford, H. A., Ghigo, F. D., Giorgini, J. D., Jao, J. S., \& Teitelbaum, L. 2015, DPS conference, 47 , abstract 402.05

Connors, M., Chodas, P., Mikkola, S., Wiegert, P., Veillet, C., \& Innanen, K. 2002, Meteoritics E Planetary Science, 37, 1435

Christou, A. A. \& Asher, D. J. 2011, MNRAS, 414, 2965

Evans, J. V. \& Hagfors, T. 1968, Radar Astronomy, MIT Lincoln Laboratory Publications, McGraw-Hill Inc., p. 53

Kwiatkowski, T., Kryszczyńska, A., Polińska, M., Buckley, D. A. H., O’Donoghue, D., Charles, P. A., Crause, L., Crawford, S., Hashimoto, Y., Kniazev, A., Loaring, N., Romero Colmenero, E., Sefako, R., Still, M., \& Vaisanen, P. 2009, A\&\&A, 495, 967 
Mainzer, A., Grav, T., Bauer, J., Conrow, T., Cutri, R. M., Dailey, J., Fowler, J., Giorgini, J., Jarrett, T., Masiero, J., Spahr, T., Statler, T., \& Wright, E. L. 2015, AJ, 149, 172

Margot, J. L. \& Nicholson, P. D. 2003, BAAS, 35, 1039

Ostro, S. J., Pravec, P., Benner, L. A. M., Hudson, R. S., Sarounova, L., Hicks, M. D., Rabinowitz, D. L., Scotti,J., V., Tholen, D. J., Wolf, M., Jurgens, R. F., Thomas, M. L., Giorgini, J. D., Chodas, P. W., Yeomans, D. K., Rose, R., Frye, R., Rosema, K. D., \& Winkler, R., Slade M. A. 1999, Science, 285, 557

Ostro, S. J. \& Giorgini, J. D. 2004, Mitigation of Hazardous Comets and Asteroids (M. J. S. Belton, T. H. Morgan, N. Samarasinha, D. K. Yeomans, eds.) Cambridge University Press, Cambridge

Pravec, P. \& Harris, A. W. 2000, Icarus, 148, 12

Quirk, K. \& Srinivasan, M. 2013, The Interplanetary Network Progress Report, 42-192, 1-46

Sánchez, P. \& Scheeres, D. J. 2014, Meteoritics \& Planetary Science, 49, 788

Scheeres, D. J., Hartzell, C. M., Schez, P., \& Swift, M., 2010, Icarus, 210, 968

Slade, M. A., Lee, C. G., Jao, J. S., Benner, L. A. M., Brozovic, M., Giorgini, J. D., \& Busch, M. W., 2010, BAAS, 42,1080

Slade, M. A., Benner, L. A. M., \& Silva, A. 2011, Proc. of IEEE, 99, 757

Wiegert, P., Connors, M., Chodas, P., Veillet, C., Mikkola, S., \& Innanen, K., 2002, Eos Trans. $A G U, \mathrm{P} 11 \mathrm{~A} 0352$ 